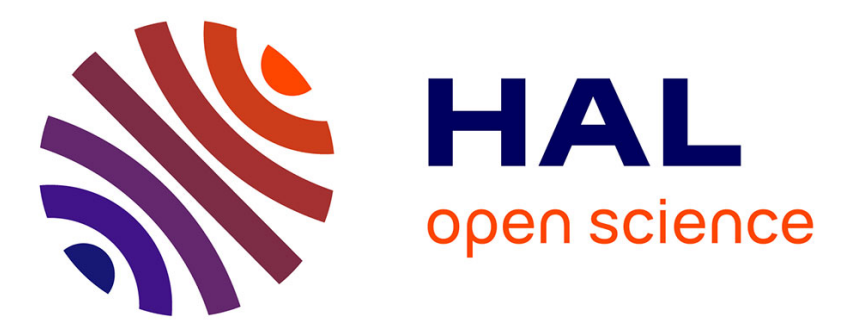

\title{
Comparing Topological and Physical Approaches to Network Modeling for the Optimization of Failure-Resilient Electrical Infrastructures
}

Yi-Ping Fang, Nicola Pedroni, Enrico Zio

\section{- To cite this version:}

Yi-Ping Fang, Nicola Pedroni, Enrico Zio. Comparing Topological and Physical Approaches to Network Modeling for the Optimization of Failure-Resilient Electrical Infrastructures. ICVRAM \& ISUMA 2014, Jul 2014, Liverpool, United Kingdom. pp.725 - 735, 10.1061/9780784413609.074. hal-01108227

\section{HAL Id: hal-01108227}

https://hal-centralesupelec.archives-ouvertes.fr/hal-01108227

Submitted on 22 Jan 2015

HAL is a multi-disciplinary open access archive for the deposit and dissemination of scientific research documents, whether they are published or not. The documents may come from teaching and research institutions in France or abroad, or from public or private research centers.
L'archive ouverte pluridisciplinaire HAL, est destinée au dépôt et à la diffusion de documents scientifiques de niveau recherche, publiés ou non, émanant des établissements d'enseignement et de recherche français ou étrangers, des laboratoires publics ou privés. 


\title{
Comparing Topological and Physical Approaches to Network Modelling for the Optimization of Failure Resilient Electrical Infrastructures
}

\author{
Yi-Ping. Fang ${ }^{1}$, Nicola Pedroni ${ }^{1}$, and Enrico Zio ${ }^{1,2}$ \\ ${ }^{1}$ Chair on Systems Science and Energetic Challenge, European Foundation for New \\ Energy - EDF, École Centrale Paris - Supelec, Paris, France \\ ${ }^{2}$ Energy Department, Politecnico di Milano, Italy
}

\begin{abstract}
Large scale outages on critical infrastructures (CIs) resulting from small initial failures, although infrequent, are increasingly disastrous to society; thus, it's imperative to optimally design these systems in order to maximize their resilience against cascading failure. The optimization task requires the understanding and modelling of the dynamics of these systems. In this paper, we consider two approaches to infrastructure modelling: (i) a simplified (computationally cheap) graph-theoretical (topological) approach based on network theory; (ii) a realistic (computationally intensive) physical approach based on power flow models. The objective of the work is to investigate whether the optimal network obtained using a simplified topological model is also physically optimal when a more realistic power flow model is embraced. This is performed through simulations on the $400 \mathrm{kV}$ French power transmission network. A discussion of the usefulness of topological models to study the dynamics of cascading failures in electrical infrastructures is also contributed.
\end{abstract}

\section{INTRODUCTION}

Our modern society has come to depend on large-scale critical infrastructures (CIs) to deliver resources and services to consumers and businesses. These CIs are complex networks of interconnected functional and structural elements. Large scale outages on these systems, although infrequent, are increasingly disastrous to society, with estimates of direct costs up to billions of dollars and inestimable indirect costs (Lewis, 2006). Research regarding modeling, prediction and mitigation of cascading failures in CIs, whereby small initial disturbances may propagate through the whole system, has tried to address the problem by different ways (Baldick et al., 2008).

Albert et al. (2004) demonstrated that the vulnerability of modern infrastructure networks is inherent to their organization. Thadakamalla et al. (2004) revealed that the topology of a supply infrastructure has great impact on its resilience. Essentially, intensive studies in the optimal design and renovation of CIs have been emerged during the last decades (Gutfraind, 2010). In practical cases, the cost of knocking down an existing infrastructure and rebuilding it from scratch is 
prohibitive. A more practicable alternative is to reconfigure parts of the system, e.g. by reallocation of the links which connect production facilities to consumers.

The primary objective of this paper is to propose a methodology for optimal allocation of the links connecting generators and distributors in a power transmission network for obtaining high resilience to cascading failures while keeping the investment costs low. Formulated as a nonlinear and combinatorial multi-objective optimization problem, this problem is solved by an evolutionary method, i.e., the non-dominated sorting binary differential evolution (NSBDE) (Li et al., 2012).

The search by the NSBDE requires also: (i) the construction of a model to describe the cascading failure process in the network, and (ii) the repeated evaluation of the model for every possible solution proposed by the algorithm during the search. With respect to the model, two approaches are typically considered in the analysis of power transmission systems: complex network theory models, such as the Motter-Lai (ML) model (Motter and Lai, 2002) and power flow models, such as the ORNLPserc-Alaska (OPA) model (Dobson et al., 2001).

The OPA model seeks to faithfully describe the dynamics of the power flows during the evolution of the cascading failure, by explicitly incorporating the standard DC power flow equations and minimizing generation cost and load shedding (Dobson et al., 2001); this results in a significant increase in the computational burden, which limits its application to large-scale realistic networks (Baldick et al., 2008). On the contrary, the ML model is relatively simple and it does not consider all the physical details of the electrical system. Its application to large-scale networks is feasible and more readily than OPA (Kinney et al., 2005). However, the abstract nature of the ML model has posed questions on whether or not it is adequate in practice, although it offers a new and interesting perspective on the study of cascading failures on power grids (Sun and Han, 2005; Cupac et al., 2013).

In the present paper, we embrace the topological ML cascading failure model and embed it in the NSBDE for optimally solving the problem of generatorsdistributors link allocation. For exemplification, we apply the method to the $400 \mathrm{kV}$ French power transmission network, under the objectives of maximizing network resilience to cascading failure and minimizing investment costs. We, then, tackle the problem of realistic significance of the results that can be obtained with the proposed methodology. For this reason, optimal power flow is performed on the optimal network topologies found.

The reminder of this paper is organized as follows. In Section 2, we introduce the ML and OPA cascading failure models briefly. We, then, formulate the multiobjective optimization taking investment costs and failure resilience into account in Section 3. Section 4 illustrates the French $400 \mathrm{kV}$ power transmission network case study and the analysis of the results. Conclusion is drawn in Section 5.

\section{CASCADING FAILURE MODELS CONSIDERED IN THIS WORK}

\subsection{The ML model}

The ML model has been proposed by Motter and Lai (2002), with extensions to differentiate generators and loads (Kinney et al., 2005). The network is 
represented as an undirected graph $Q$ with a set of $N$ nodes representing $N_{G}$ generation nodes and $N_{D}$ loading nodes, interconnected by a set of edges representing transmission lines. The ML model assumes that at each time step, one unit of the relevant quantity is exchanged between every pair of generation and loading nodes, and transmitted along the shortest path connecting them. The flow at one node is, then, the number of shortest paths passing through it. More precisely, the load $L_{k}$ of node $k$ is quantified by the node betweenness:

$$
L_{k}=\frac{1}{N_{G} N_{D}} \sum_{i \in V_{G}, j \in V_{D}, i \neq j \neq k} \frac{n_{i j}(k)}{n_{i j}}
$$

where $n_{i j}$ is the number of shortest paths between generator nodes and distributor nodes, and $n_{i j}(k)$ is the number of generator-distributor shortest paths passing though node $k$.

The capacity of node $k$ is assumed to be proportional to its initial node $L_{k}$ with a network tolerance parameter $\alpha$,

$$
C_{k}=(1+\alpha) L_{k}
$$

The concept of the tolerance parameter $\alpha(\alpha>0)$ can be understood as an operating margin allowing safe operation of the component under potential load increment. The occurrence of a cascading failure is initiated by removal of a node, which in general changes the distribution of shortest paths. Then the load at a particular node can change and if it increases and exceeds its capacity, the corresponding node fails. Any failure leads to a new redistribution of loads and, as a result, subsequent failures can occur.

The vulnerability of network $Q$ against cascading is characterized by the fraction of network efficiency lost in the cascading failure:

$$
\operatorname{Vul}(Q)=\frac{E(Q)-E(\bar{Q})}{E(Q)}
$$

where $\operatorname{Vul}(Q) \in(0,1)$ and $\bar{Q}$ represents the residual network structure after the cascading failure. $E(Q)$ measures the network efficiency based on the node pair shortest path distance between generators and distributors:

$$
E(Q)=\frac{1}{N_{G} N_{D}} \sum_{i \in V_{G}} \sum_{j \in V_{D}} \frac{1}{d(i, j)}
$$

where $d(i, j)$ is the number of edges for an unweighted network or the sum of edge weights for a weighted network in the shortest path from $i$ to $j$.

Complex network theory models, such as the ML that we use within our optimization framework in Section 3, have no direct physical relation to the mechanisms of realistic power grids, but they have the key advantage that by utilizing techniques from graph theory they can be applied to analyze various largescale networks. For this reason, this modeling approach is seeing increasing applications for modelling cascading failure in infrastructure networks.

\subsection{The OPA model}

The OPA model (Dobson et al., 2001) contains two different time scale dynamics, i.e., fast power flow dynamics and slow power grid growth dynamics, and describes the complexity and criticality of power systems. Our analyses focus on the fast power flow dynamics, in order to ensure comparability with the ML model shortest path assumption. 
The cascading failure model is based on the standard DC power flow equation,

$$
F=A P
$$

where $F$ is a vector whose components are the power flows through the lines, $P$ is a vector whose components are the power injection of each node, and $A$ is a constant matrix that depends on the network structure and impedances (see Donbson et al., 2001 for details about the computation of $A$ ).

The generator power dispatch is solved using standard linear programming methods. Using the input power demand, the power flow equation (5) is solved with the condition of minimizing the following cost function:

$$
\text { Cost }=\sum_{i \in V_{G}} P_{i}(t)+K \sum_{j \in V_{D}} P_{j}(t)
$$

where $V_{G}$ and $V_{D}$ are the sets of generators and distributors, respectively. This definition gives preference to generation shift whilst assigning a high cost (set $K=$ 100) to load shedding, and it is assumed that all generators operate at the same cost and that all loads are served with equal priority. The minimization is done with the following constraints:

(1) Generator power injections are generally positive and limited by installed capacity limits: $0 \leq P_{i} \leq P_{i}^{\max }, i \in V_{G}$.

(2) Loads always have negative power injections: $P_{j}^{\text {dem }} \leq P_{j} \leq 0, j \in V_{D}$.

(3) The absolute flow through links is limited by link capacities: $\left|F_{i j}\right| \leq F_{\text {max }}$.

(4) Total power generation and consumption remain balanced: $\sum_{i \in V_{G} \cup V_{D}} P_{i}=0$.

After solving the linear optimization, a link is considered to be overloaded if the power flow through it is within $1 \%$ of its limit capacity $F_{\text {max }}$. Each overloaded line may outage with probability $p_{1}$. If an overloaded line experiences an outage, its power flow limit $F_{\max }$ is divided by a very large number $k_{1}$ to ensure that practically no power may flow through the line. Besides, to avoid a matrix singularity, the impedances of failed lines are multiplied by a large number $k_{2}$, resulting in changes of the network matrix $A$.

Load shedding is utilized to quantify the damage of the cascading failure. For an individual node, load shedding is defined as the variance between its power injection and demand:

$$
S_{i}=P_{i}^{\text {dem }}-P_{i}
$$

Subsequently, total load shedding for the system is:

$$
S=\sum_{i \in V_{D}} S_{i}
$$

Finally, system load shedding is normalized by its total demand $D$ and used as a measure of cascading failure damage:

$$
S / D=\frac{\sum_{i \in V_{D}} S_{i}}{\sum_{i \in V_{D}} P_{i}^{d e m}}
$$

The fact that simulation results from OPA model are consistent with historical blackout data for real power systems has justified its effectiveness (Carreras et al., 2004). However, the applications of OPA have generally been limited to networks with a relatively small number of nodes compared to real power grids (Sun and Han, 2005), due to the computational efforts involved.

\section{OPTMIZATION MODEL}


For a given network, cascading failure resilience could be enhanced in many ways. In this paper, we focus on choosing the connection patterns between generators and distributors of a realistic power grid, so as to optimize its resilience to cascading failures. Given the goal of analyzing a realistic-size network, the ML cascading failure model is used to evaluate the resilience of a connection pattern. By associating a cost to each link posed in the network, the optimization also seeks to minimize the total cost.

The network is modeled as a weighted graph, in which the edge weights are given by their physical distances which we assume directly related to the transmitting cost of the link. We define the variables to be optimized as the links of generation nodes to the different distribution nodes:

$$
X_{i j}=\left\{\begin{array}{c}
1, \text { if } i \text { is connected with } j \text { directly } \\
0, \text { otherwise }
\end{array}\right.
$$

for all $i \in V_{G}$ and $j \in V_{D}$. Two constraints have to be met when rewiring generators and distributors: (1) each distributor node is required to connect with at least one generator node or other distributor node, to make it accessible to the power supplying generators; (2) each generator node has to connect at least with one distributor node.

We assume that the cost associated with each connection cutting and rewiring is linearly proportional to the physical length of the link, with factor $\varphi$. The total investment cost of a reconstructed pattern $X$ in the power transmission network can be defined as

$$
C=\sum_{i \in V_{G} j \in V_{D}} \varphi X_{i j} d(i, j)
$$

where $d(i, j)$ is the physical distance between $i$ and $j$.

The cascading failure resilience of each reconstructed pattern $X$ can be quantified by the vulnerability of the new network, given by equation (3). It should be noted that the effect of the type of initial event could significantly influence the cascading failure result: the efficiency loss of a cascade triggered by the failure of a critical component could be much more severe than that originated by the failure of a normal component. Therefore, we consider a worst-case scenario in this study by choosing the failure of one of the top five most loaded (largest betweenness) nodes as initial failure in each cascade process simulation and, then, the results are averaged on the number of simulations.

Through the quantification of the connection pattern cost and cascading failure vulnerability, the facility allocation problem is formulated as a multi-objective optimization problem:

$$
\begin{aligned}
&\left\{\begin{array}{l}
\min C\left(X_{i j}\right) \\
\min V u l\left(Q_{X_{i j}}\right)
\end{array}\right. \\
& \text { s.t. }\left\{\begin{array}{l}
\sum_{i \in V_{G} \cup V_{D}} X_{i j}>0 \forall j \in V_{D} \\
\sum_{j \in V_{D}} X_{i j}>0 \forall i \in V_{G}
\end{array}\right.
\end{aligned}
$$

The objective function (12a) is the sum of the fixed rewiring costs; (12b) expresses the resilience objective. The two constraints mentioned above are enforced by formulas (12c) and (12d), respectively. Observe that the least costly generator allocation is simply that with no links among facilities and consumers. 
In our work, the multi-objective optimization problem $(12 \mathrm{a})-(12 \mathrm{~d})$ is tackled by the Non-dominated Sorting Binary Differential Evolution (NSBDE) algorithm. Readers could refer to Fang et al. (2013) for the details about this optimization algorithm.

\section{CASE STUDY AND RESULTS ANALYSIS}

\subsection{Case study and parameters setting}

In this paper, the $400 \mathrm{kV}$ French power transmission network (FPTN400) (RTE, 2011) is taken for exemplification of the proposed approach. It has 171 substations and 220 transmission lines (Figure 1). Among these substations, 26 are generators and 145 are distributors. For reallocation of the power generating nodes to the other nodes, the NSBDE algorithm is applied. The network tolerance parameter $\alpha$ is set to 0.3 to simulate the normal operating condition; linkage cost parameter $\varphi$ is set to 1 .

At the beginning of the simulation, all 55 links among generators and distributors in the FPTN400 are cut off. The population is initialized by randomly assigning 0 or 1 to each bit of each chromosome in the population, forming a group of potential solutions. For evaluating the cascading vulnerability of a given connectivity pattern, the ML cascading failure model is run starting from failing one of the top five most loaded nodes in repeated cascading simulations at the end of which the vulnerability values are averaged.

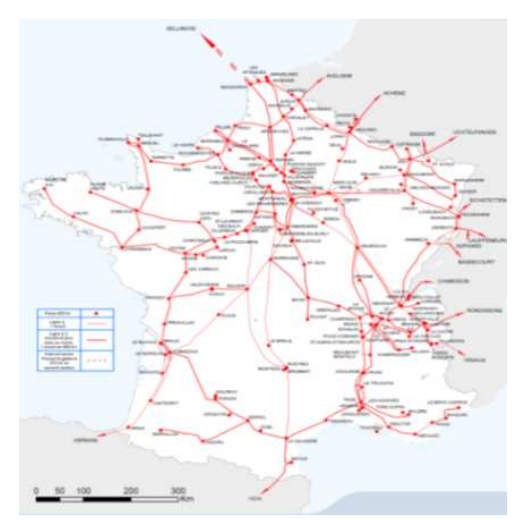

Figure 1. The 400kV French power transmission network

\subsection{Topological optimization results}

The Pareto front obtained by the NSBDE algorithm at convergence is shown in Figure 2(a). The diamond point represents the present network with the existing connection which is also the least costly network; the square point is the most resilient solution, whose cascading vulnerability is 0.184 . It is not unexpected that the original network is the least costly one, since the electrical transmission lines and substations are placed with geographical constraints and connections between two distant substations are avoided. Truly, cost-efficiency is a major consideration in building power transmission networks. 
It is also noted from Figure 2(a) that the cascading resilience of the FPTN400 can be improved significantly, though at a cost; the network vulnerability is decreased from 0.728 to 0.184 (when $\alpha=1.3$ ) with an increased cost of $7.3 \times 10^{3}$. Figure 2(b) reports the cascading vulnerability comparison between the original network and the most resilient one (Pareto solution \#17) with different tolerance parameters. It shows that when the network tolerance is very low, i.e. $0<\alpha<0.1$, the optimized network loses most of its efficiency, i.e., it is quite vulnerable to intentional attacks possibly due to its intensive loading condition. However, when $\alpha$ $\geqslant 0.3$ (which is generally the normal operating condition (Baldick et al., 2008)), the optimized network loses less than $20 \%$ of its efficiency during a cascading failure initiated by intentional attack.
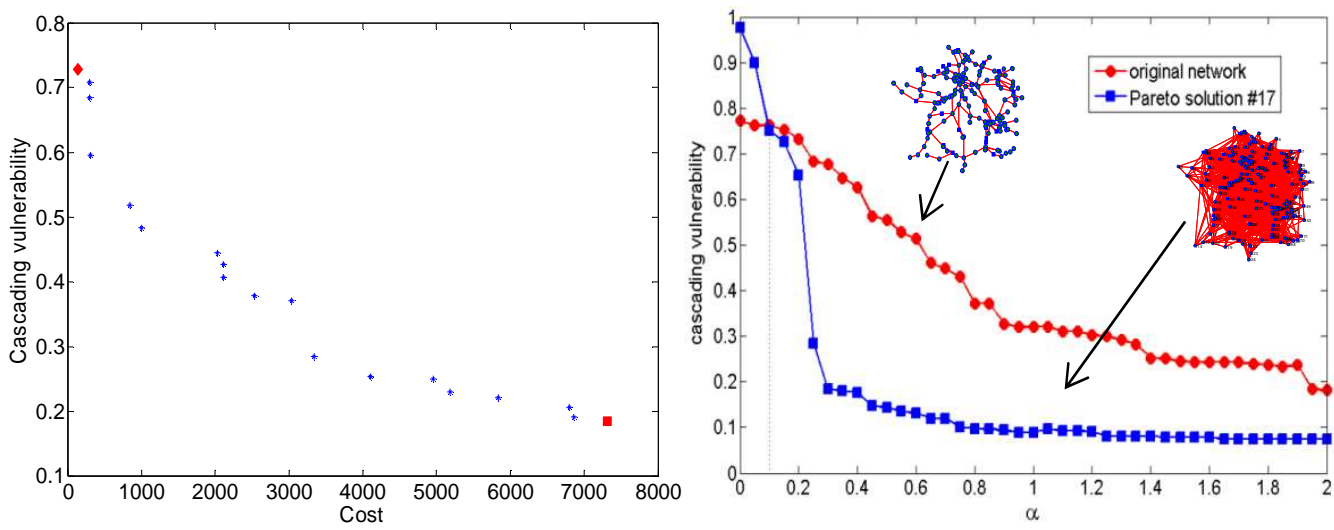

Figure 2. (a) Pareto front reached by a population of 25 chromosomes evolving for 300 generations; (b) Comparison of the cascading vulnerability between the original and the most resilient networks under different network tolerance values

Albeit a substantial improvement of the cascading failure resilience of the FPTN400 is possible by adding redundant links, a tradeoff between the cost and resilience improvement is necessary for rational decision-making. Along the Pareto frontier of the potential solutions, there are some points at which a small sacrifice of cost gives a large gain of cascading resilience. More generally, by taking a network solution and its neighbor on the frontier (the less costly one), one can define a rate of change of cascading resilience with respect to cost: $|\Delta V u l / \Delta \operatorname{cost}|$. This rate can be utilized as a reference to choose the optimized network: the larger the ratio, the more preferred the network is.

Figure 3 reports the topology of the network corresponding to the Pareto solution \#3 $(310.6,0.59)$ whose $|\Delta V u l / \Delta \operatorname{cost}|$ value is comparatively large. The bold links denote the 10 added links with respect to the original real network: notice that only 10 links are required to be rewired for the original network to gain a $19.2 \%$ cascading resilience improvement (the cascading vulnerability is decreased from 0.73 to 0.59 ). Besides, it is noted from Figure 3 that the newly added links tend to connect distant generator and distributor pairs, indicating that the installation of power lines between remote power substations can improve the resilience of the system, although at larger costs. 


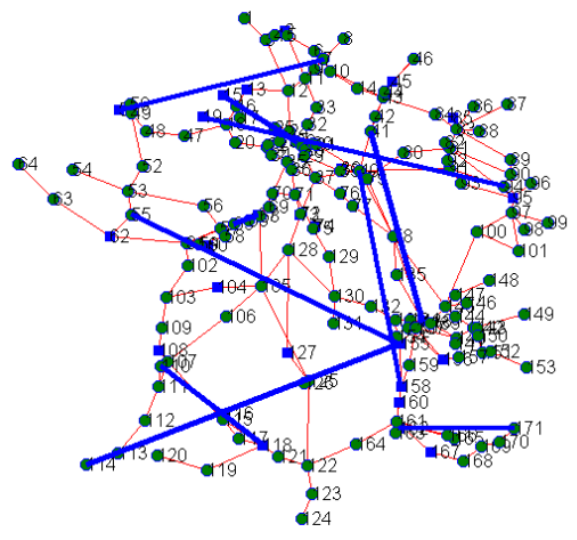

Figure 3. The topology of the Pareto solution \#3

\subsection{Validation by the OPA model}

In this section, the more realistic OPA model introduced in Section 2.2 is utilized to verify the optimization results based on the abstract ML model. The verification is not straightforward due to the differences of the two models in the way of representing system capacity, in the iterative algorithms they rely on, and in the way of measuring the damage. Accordingly, some assumptions and adjustments to the OPA model are necessary to ensure its applicability to assess the optimization solutions obtained based on the ML model.

\subsubsection{OPA Adjustments}

To facilitate comparability with the ML model, all the generators are assumed to have equal capacity, and all the loads are assumed to have equal constant demand (we use 26, i.e. the number of generators in the simulation). Furthermore, all edge impedances are calculated using the typical reactance value $0.28 \mathrm{ohm} / \mathrm{km}$ at $50 \mathrm{~Hz}$ (Zhou and Bialek, 2005). This heterogeneous impedance setting aligns with the weighted edge initialization in the ML model.

A simple initialization strategy (Cupac et al., 2013) is utilized to set the link capacity in the OPA model. In particular, the values of the initial flows $\overline{F_{i j}}(0)$ and of the link capacities $F_{i j}^{\max }$ are determined as follows: demand for all distributor nodes is fixed to a constant amount, as mention above, and total generation capacity is set to be equal to total demand, and equally divided among the generators. Then, the power flows along the lines are estimated by assuming that every distributor node would obtain an equal amount of power from every generator. The initial flows are calculated by selecting a generator (one at a time), setting all other generator capacities to 0 and then computing power flows to each distributor node. The sum of the power flows over all the generators results in the estimated initial flow along each link, $\overline{F_{i j}}(0)$. Analogous to the initialization process in the ML model, the maximum capacity for a link connecting nodes $i$ and $j$ is given by

$$
F_{i j}^{\max }=(1+\alpha)\left|\overline{F_{i j}}(0)\right|
$$


It is noted that the values of the initial flows $\overline{F_{i j}}(0)$ are only used to set the link flow capacities $F_{i j}^{\max }$ in such a way that they are comparable to the capacities $C_{k}$ used by the ML model. The network tolerance parameter is set to $0 \leq \alpha \leq 2$ in our approach, parallel to the ML model, representing excess transmission capacity. Then, the node transmission capacity is modelled as the sum of link flow capacities of adjacent links $\sum_{j \in V_{j}} F_{i j}^{\max }$ where $V_{j}$ is the set of nodes directly connected to node $i$.

In the OPA implementation, the probability of an overloaded link is set to $p_{1}=1$, where an overloaded node fails and is removed from the network with certainty. Besides, the cascade is initiated in the same manner in the ML model, as stated in Section 3.

\subsubsection{Evaluation Results}

Three representative solutions (i.e., the least cost network FPTN400, the most resilient network, namely, solution \#17, and Pareto solution \#3 whose $|\Delta V u l / \Delta \operatorname{cost}|$ value is comparatively large) along the Pareto front are chosen as the basic network topologies to be verified by the OPA model. The calculation of the correlation coefficients between capacities $C_{k}$ and $F_{i j}^{\max }$ under ML and OPA models shows that node capacities in ML are highly correlated with node capacities in OPA (the correlation coefficients are $0.904,0.890$ and 0.619 for FPTN400, Pareto solution \#3 and Pareto solution \#17, respectively).

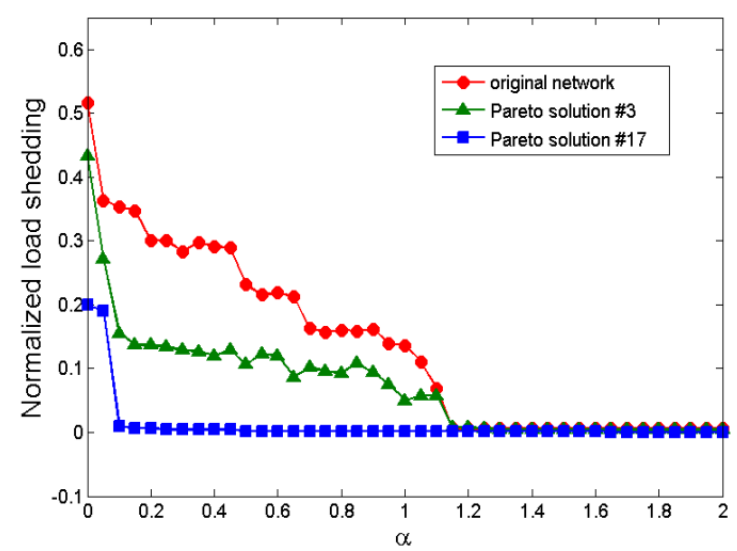

Figure 4. Cascading vulnerability (normalized load shedding) evaluated by the OPA model for the three chosen networks over a range of network tolerance values $\alpha$.

In Figure 4, we plot the curves of normalized load shedding $S / D$ versus network tolerance $\alpha$ obtained by applying the OPA model to the three representative networks selected from the Pareto front. Analogous to the ML model (Figure 2(b)), the network damages decreases when network tolerance increases for all the networks. When network tolerance value is high enough $(\alpha>1.2)$, any small intentional disturbance on the network would tend to cause quite low damage to the functioning of the network $(<1 \%)$. Most importantly, it is observed that in the OPA simulation, the network corresponding to Pareto solution \#3 (green triangle curve) is 
more resilient, i.e., it presents less load shedding than the original network (circle curve) over a wide range of network tolerance $\alpha$ (i.e., $0<\alpha<1.2$ ); in addition, Pareto solution \#7 (which is the most resilient network according to the ML model) presents the lowest load shedding among the three networks over the entire range of $\alpha$ values considered. This ranking of cascading failure resilience is consistent with the simulation results based on ML model, verifying that the insights gained by the topological optimization approach are valid.

Also important is to remember that the results produced by the simple ML topological model are obtained at a much lower computational cost than those of the OPA model: actually, the average time needed to carry out a single cascading failure simulation is $3.9 \mathrm{~s}$ and $20.8 \mathrm{~s}$ for the ML and OPA models, respectively, on a double $2.4 \mathrm{GHz}$ Intel CPU and 4 GB RAM computer.

\section{CONCLUSION}

In this paper, we have investigated the allocation of generators to distributor nodes by rewiring links under the objectives of maximizing the network cascading failure resilience and minimizing the investment costs. The NSBDE algorithm is applied within a Pareto optimality scheme of search for non-dominated solutions. To simulate and quantify the cascading failure resilience of potential connection pattern selected during the NSBDE search, a complex network model - the ML model - has been used, to exploit its rapidity of calculation. The results of the case study have shown that the cascading resilience of a realistic power transmission network can be improved at an acceptable cost.

To validate the physical significance of the topological optimization results, a more realistic power flow model - the OPA model - has been considered. The OPA model has been applied to three solutions selected from the Pareto front found by the topological optimization. The ranking of the three selected networks with respect to their vulnerability to targeted attacks is consistent with that of the ML model; in addition, the computational time required by the ML approach is shown to be 5.5 times lower than that of the OPA approach. This verifies (i) the physical meaningfulness of the topological optimization solutions and (ii) the practical usefulness of abstract cascading models in network optimization tasks.

\section{REFERENCES}

Albert, R., Albert, I., and Nakarado, G. L. (2004). "Structural vulnerability of the North American power grid.” Physical Review E 69.2: 025103.

Baldick R., et al. (2008). "Initial review of methods for cascading failure analysis in electric power transmission systems IEEE PES CAMS task force on understanding, prediction, mitigation and restoration of cascading failures." Power and Energy Society General Meeting-Conversion and Delivery of Electrical Energy in the 21st Century, IEEE, pp. 1-8.

Carreras B. A., Newman D. E., Dobson I., and Poole A. B. (2004). "Evidence for self-organized criticality in a time series of electric power system blackouts." 
Circuits and Systems I: Regular Papers, IEEE Transactions on, 51.9: 17331740.

Cupac V., Lizier J. T., Prokopenko M. (2013). "Comparing dynamics of cascading failures between network-centric and power flow models." International Journal of Electrical Power \& Energy Systems, 49: 369-379.

Dobson I., Carreras B. A., Lynch V. E., and Newman D. E. (2001) "An initial model for complex dynamics in electric power system blackouts." In: Proceedings of the 34th annual Hawaii international conference on system sciences, pp. 51.

Fang, Y.-P., Pedroni, N. and Zio, E. (September 2013). "Optimal Production Facility Allocation for Failure Resilient Critical Infrastructures." Safety, Reliability and Risk Analysis: Beyond the Horizon, In Proceedings of the ESREL 2013 Conference, Amsterdam.

Gutfraind A. (2010). "Optimizing topological cascade resilience based on the structure of terrorist networks." PloS one 5.11: e13448.

Kinney R., et al. (2005). "Modeling cascading failures in the North American power grid." The European Physical Journal B-Condensed Matter and Complex Systems, 46.1: 101-107.

Lewis, T. G. (2006). "Critical infrastructure protection in homeland security: defending a networked nation." Wiley. com.

Li Y.-F., Sansavini G., and Zio E. (2012). "Non-Dominated Sorting Binary Differential Evolution for the Multi Objective Optimization of Cascading Failures Protection in Complex Networks." Reliability Engineering \& System Safety.

Motter A. E., and Lai Y.-C. (2002). "Cascade-based attacks on complex networks." Physical Review E 66.6: 065102.

RTE. (2011). "Le Réseau de Transport d'Electricité 400 kV." http://www.rtefrance.com.

Sun K., and Han Z.-X. (2005). "Analysis and comparison on several kinds of models of cascading failure in power system." In: Transmission and distribution conference and exhibition: Asia and Pacific, 2005 IEEE/PES, p. 1-7.

Thadakamaila, H. P., et al. (2004). "Survivability of multiagent-based supply networks: a topological perspective." Intelligent Systems, IEEE 19.5: 24-31.

Zhou Q., and Bialek J. W. (2005). "Approximate model of European interconnected system as a benchmark system to study effects of cross-border trades." Power Systems, IEEE Transactions on, 20.2: 782-788. 